\title{
PAN 11347
}

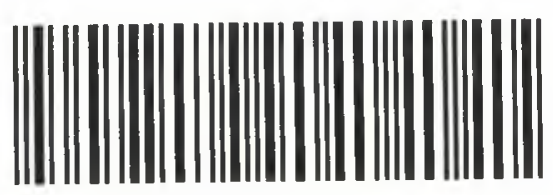

(Aus dem physiologischen Institut der Universität Lemberg.)

\section{Physiologische Untersuchungen,} betr. Reflexbahnen in der grauen Substanz des Rückenmarks.

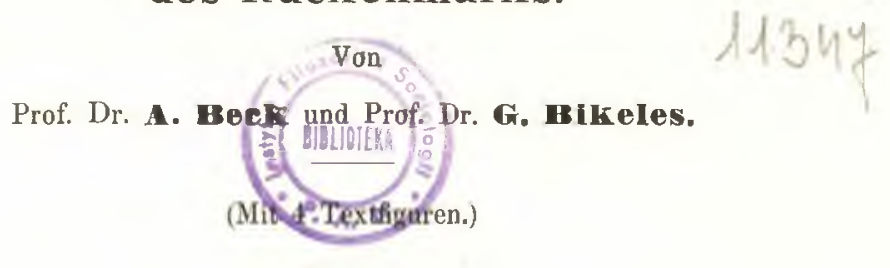

Bekanntlich ziehen am Rückenmarksquerschnitt viele Hinterstrangskollateralen, in der Gegend der Basis des Hinterhorns zu einem Bündel vereinigt, im Vorderhorn fächerförmig ausgebreitet, bis hart an die motorischen Zellen heran (Fig. 1a). Man nennt diese längsten Hinterstrangskollateralen auch Reflexkollateralen ${ }^{1}$ ) und stellt sich vor, dass gerade diese hart an die motorischen Zellen heranreichenden sensiblen Fasern am besten zur Reizübertragung auf motorische Elemente behufs Auslösung des Reflexes geeignet seien. Tatsächlich findet sich hier - wie nirgends mehr im ganzen Zentralnervensystem - das physiologische Schema eines Reflexbogens wie am augenscheinlichsten dargestellt: die von der Peripherie herkommende zentripetale Nervenfaser stösst fast unmittelbar auf eine motorische Zelle, deren zentrifugaler Fortsatz wiederum direkt zum Muskel hinzieht.

Wie verlockend es aber wäre, aus diesem anatomischen Bilde Schlüsse über die physiologische Tätigkeit zu ziehen, dürfen doch

1) Reflexkollateralen schlechtweg werden ausschliesslich die eben beschriebenen längsten an die motorischen Zellen heranreichenden Kollateralen genannt. Ist eine physiologische Bezeichnung für anatomische Verhältnisse überhaupt nicht wünschenswert, so ist die noch verallgemeinernde Benennung "Reflexkollateralen" auch für die kurzen Kollateralen, wie dies Langendorff in $\mathrm{Nagel}$ 's Handb. Bd. 4 Hälfte I S. 284 tut, ganz zu verwerfen, da die Hinterstrangskollateralen und vielleicht überwiegend gerade die kurzen (Fig. 1 b) für allerlei Sensibilitätsleitung - ausser für Reflexe - in Betracht kommen. 
blos aus der Kenntnis von morphologischen Verhältnissen im Zentralnervensystem keine Schlüsse auf die Funktion gezogen werden. Es war daher die Frage sehr berechtigt, ob diese langen Hinterstrangkollateralen wirklich für das Zustandekommen des Reflexes

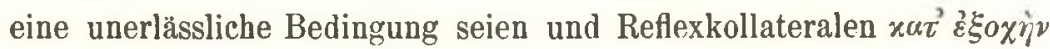
darstellen.

Zur Beantwortung dieser Frage wurde ein, beim Hund wie beim Menschen konstanter, auf leichteste Reizung (Berührung, minimalen Stich) erfolgender Hautreflex, nämlich die reflektorische Zehenbeugung, erhältlich bei Reizung der Sohlenhaut, gewählt.

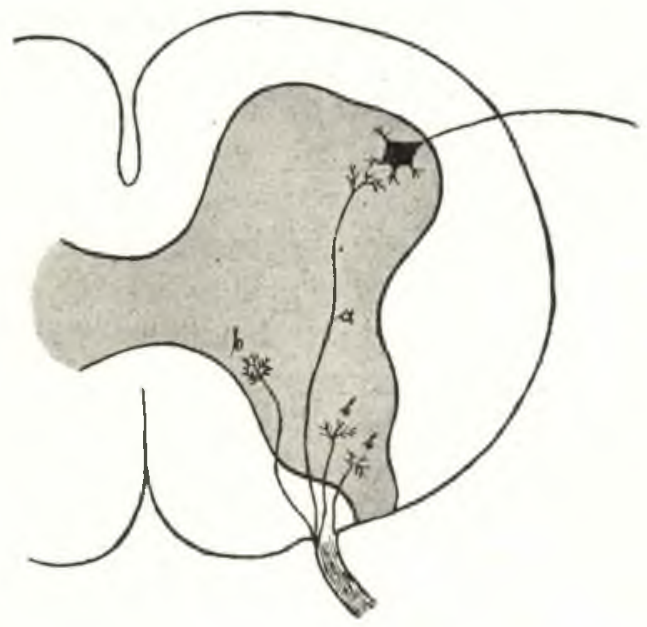

Fig. 1. $a$ lange Kollaterale. $b$ kurze im Hinterhorn verbleibende Kollateralen.

Ausser Konstanz und leichter Erhaltbarkeit ist der erwähnte Reflex auch ein segmentärer ${ }^{1}$ ) Reflex - allgemein Lokal-, Niveau- oder Etagenreflex genannt -, d. h. die zuführenden und abführenden Fasern des Reflexbogens gehören als hintere und vordere Wurzeln denselben Rückenmarkssegmenten an. Aus vorherigen Versuchen ${ }^{2}$ ) wussten wir nämlich, dass der zentripetale Teil dieses Reflexbogens seine Bezüge erhält auf dem Wege der VI. + VII. lumbalen und der

1) Viele Autoren bezeichnen die reflektorische Zehenbeugung nach Reizung der Planta (wie überhaupt die Hautreflexe) beim Menschen als cerebrale Reflexe. Beim Hunde trifft dies sicherlich nicht zu. Gerade nach Rückenmarksdurchschneidung treten am Hintertier alle Hautreflexe aufs deutlichste auf.

2) Bikeles und Gizelt, Pflüger's Arch. Bd. 106 S. 43. 
I. eventuell auch II. sakralen hinteren Wurzeln. Für den motorischen Teil wiederum kommen bezüglich der reflektorischen Zehenbeu@ung ebenfalls die VI. + VII. lumbalen und die I. eventuell auch II. sakralen vorderen Wurzeln in Betracht.

Ausgangspunkt und Basis unserer Versuche war die von einem von uns $^{1}$ ) zuerst festgestellte Tatsache, wonach von den einer hinteren Wurzel entspringenden Hinterstrangskollateralen die längsten (d. i. durch das Vorderhorn bis an die motorischen Zellen heranreichenden) demselben oder noch dem nächstfolgenden Rückenmarkssegment angehören, während weitere in grösserer Entfernung vor der Eintrittshöhe abgehende Hinterstrangskollateralen dieser hinteren Wurzel in das Vorderhorn nicht mehr eintreten. Darauf fussend wurde folgendes Verfahren eingeschlagen: Es wurde bei Hunden auf einer Seite ausnahmslos bei allen Versuchen die II. + III. sakrale Wurzel und ebenfalls die VII. lumbale Wurzel gänzlich (d. i. die vordere und die hintere Wurzel zusammen) durchschnitten. Von der I. sakralen und VI. lumbalen Wurzel dieser Seite wurde je eine vordere und je eine hintere durchschnitten (d. h. entweder die I. sakrale hintere und die VI. lumbale vordere oder umgekehrt, die I. sakrale vordere und die VI. lumbale hintere) ${ }^{2}$ ). Auf diese Weise verblieb für den in Rede stehenden Reflex nur eine einzige hintere und eine einzige vordere Wurzel, die voneinander durch ein auf dieser Seite seiner Wurzeln beraubtes Segment getrennt blieben. Die in das Vorderhorn hineinreichenden Hinterstrangskollateralen waren somit für die Vermittlung dieses Reflexes ausgeschaltet.

Um bezüglich des Reflexes gar keinen Zweifel aufkommen zu lassen, wurde das Rückenmark im Dorsalteil durchschnitten; auch waren dann die Reflexerscheinungen deutlicher und lebhafter. Von dreizehn hier verwerteten Versuchen wurden elf aseptisch operiert und die Hunde durch mehrere Tage beobachtet; nur bei zweien war die Operation nicht aseptisch und dementsprechend auch die Beobachtungsdauer eine ganz kurze. Diese Versuche ergaben nun in Kürze folgendes: In sieben Versuchen mit Erhaltensein von den für diesen Reflex in Betracht kommenden Wurzeln bloss der

1) Bikeles, Abhandl. d. Akad. d. Wissensch. zu Krakau Bd. 36. 1898, und Zentralbl. für Physiol. Bd. 12. 1898; später auch von La a insky bestätigt. Arch. für Psychiatrie Bd. 42. 1907.

2) Meist wurde noch die V. lumbale Wurzel ganz (vordere und hintere) durchschnitten. 
I. sakralen hinteren und der VI. lumbalen vorderen (aufsteigende Richtung des Reflexbogens; siehe Fig. 2) war bei vieren die reflektorische Zehenbeugung erhalten ${ }^{1}$ ); in einem Versuch fehlte $\mathrm{zwar}$ die reflektorische Zehenbeugung, dafür liess sich aber die Anwesenheit

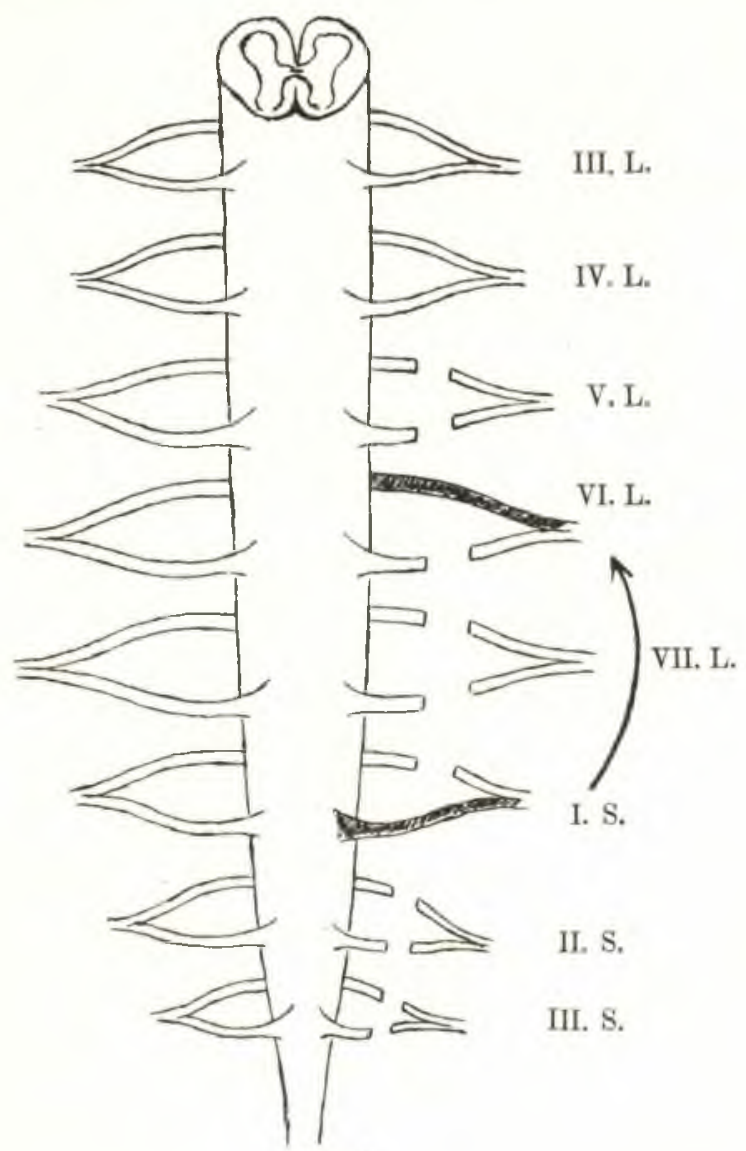

Fig. 2.

einer reflektorischen Dorsalflexion im Sprunggelenk konstatieren. (Diese Reflexerscheinung erfolgt häufig zusammen mit der reflektorischen Zehenbeugung auf Reizung derselben Stelle an der Planta.) Zwei Versuche ergaben ein negatives Resultat.

1) In zwei von diesen Fällen, in denen auf der Seite mit durchschnittenen lumbo-sakralen Wurzeln auch die V. lumbale Wurzel gänzlich $(v+h)$ durchtrennt war, waren die Reflexe auf beiden Seiten gleich lebhaft (ausdrücklich in den Versuchsprotokollen angegeben). 
In sechs Versuchen mit Intaktbleiben von den für den genannten Reflex in Betracht kommenden Wurzeln bloss der I. sakralen vorderen und der VI. lumbalen hinteren Wurzel (absteigende Richtung des Reflexbogens; siehe Fig. 3) war bei vieren reflektorische Zehen-

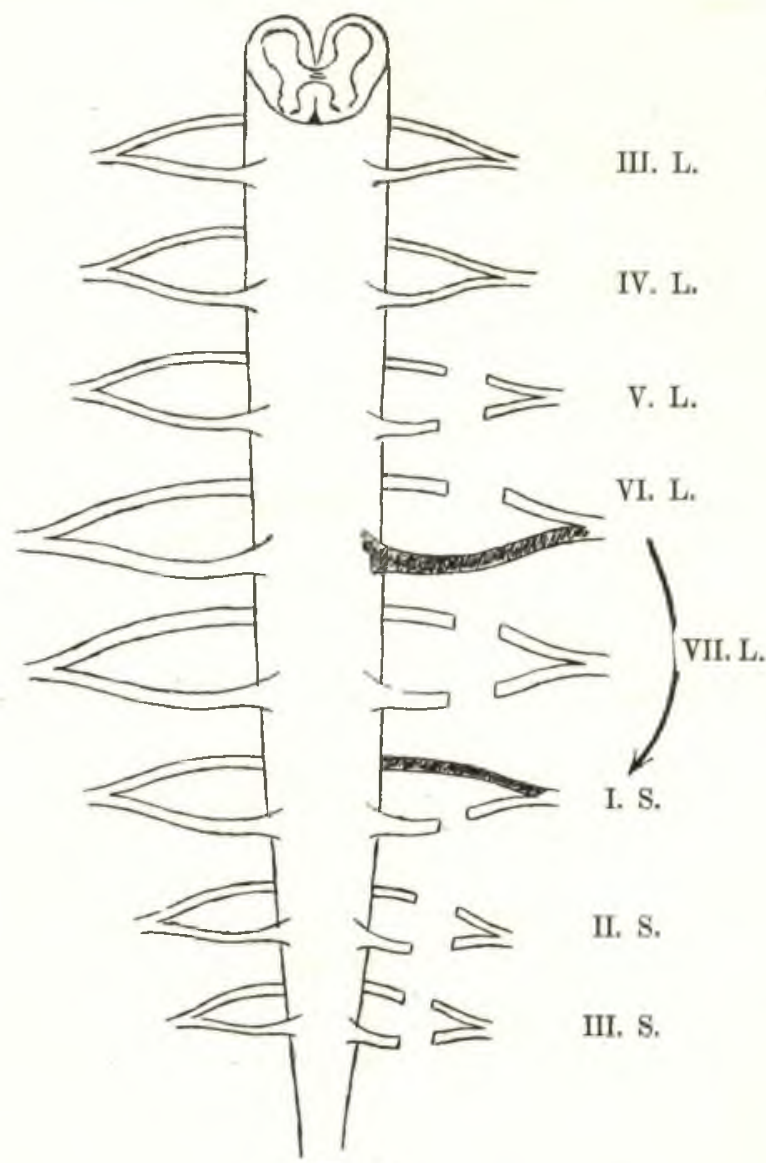

Fig. 3.

beugung vorhanden; in einem Versuch ein negatives Resultat ${ }^{1}$ ) und ein Versuch zweifelhaft.

Aus diesen Versuchen folgt also, dass auch bei einem typisch

1) In Fällen, welche negatives Resultat ergaben, rief auch die elektrische Reizung der Pfote selbst mittels starken Induktionsstromes keine Verkürzungsreflexe hervor. Wahrscheinlich hatte also auch die nicht durchschnittene hintere Wurzel durch die Operation gelitten.

E. Pflūger, Archì fūr Physiologie. Bd. 129. 
segmentären ${ }^{1}$ ) Rückenmarkreflex, erhältlich durch einen minimalen Reiz, das Zustandekommen des Reflexes sowohl in auf-, wie auch in absteigender Richtung nicht einzig von der Vermittlung seitens der an die motorischen Zellen heranreichenden Hinterstrangskollateralen abhängt.

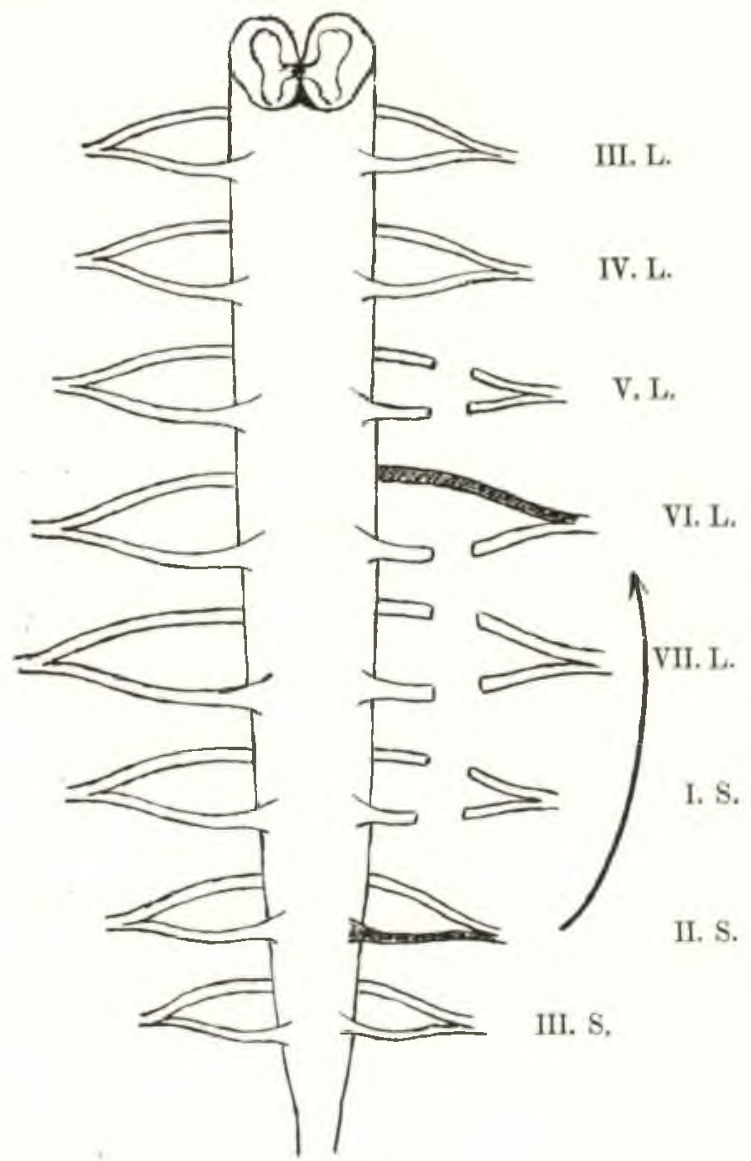

Fig, 4 .

Wir waren auch bestrebt, die Entfernung zwischen der zentripetalen und zentrifugalen Wurzel noch um ein weiteres Segment zu vergrössern, und zwar beabsichtigten wir zunächst in einigen Ver-

1) Diese Schlussfolgerung ist selbst für diejenigen, die auch beim Tier alle Hautreflexe als cerebrale ansehen wollen, stichhaltig, da nach Durchschneidung des Rückenmarks (was bei unseren Versuchen in der Regel stattfand) doch nur die spinalen Reflexbahnen in Erwägung kommen können. 
suchen für die reflektorische Zehenbeugung statt eines Reflexbogens von der I. sakralen hinteren zur VI. lumbalen vorderen Wurzel oder umgekehrt durch entsprechende Wurzeldurchschneidungen einen Reflexbogen von der II. sakralen hinteren zur VI. lumbalen vorderen oder umgekehrt von der VI. hinteren lumbalen zur II. vorderen sakralen Wurzel zu erhalten. Allein diese Versuche misslangen, wahrscheinlich schon deshalb, weil die zarte II. sakrale Wurzel bei der teilweisen Durchschneidung leicht in toto leidet. Wir schlugen daher ein anderes zum selben Resultat führendes Verfahren ein. Bekanntlich erfolgt auf Reizung (Streichen oder sehr leichten Stich) an der Planta nebst oder an Stelle einer reflektorischen Zehenbeugung auch eine reflektorische Dorsalflexion im Sprunggelenke (beim Menschen wie beim Hunde). Die reflektorische Dorsalflexion bei Reizung der Planta ist ebenfalls im grossen und ganzen als ein segmentärer Reflex anzusehen. Dessen zentrifugaler Schenkel (als zum Peroneus gehörend) verläuft beim Hund in den vorderen Wurzeln des VI. + VII. Lumbalsegmentes, ausnahmsweise auch des I. Sakralsegmentes, während der zentripetale Schenkel vom VI. lumbalen bis zum I. eventuell auch II. sakralen Segment hinabreicht ${ }^{1}$ ). Wir durchschnitten daher bei einem Hunde mit im Dorsalteil durchtrenntem Rückenmark auf der einen Seite die I. sakrale + VII. + V. lumbalen Wurzeln gänzlich (d. i. vordere und hintere); von der VI. lumbalen Wurzel wurde nur die hintere durchschnitten, während die II. und III. sakralen Wurzeln intakt blieben (Fig. 4). Bei einem solchen Versuchstier ist - falls die zweite sakrale Wurzel am Plexus lumbo-sacralis beteiligt ist - für eine reflektorische Zehenbeugung in dem II. Sakralsegment ein vollständiger Reflexbogen enthalten; bezüglich einer reflektorischen Dorsalflexion im Sprunggelenke hingegen kann von der II. sakralen Wurzel nur die hintere in Betracht kommen, während der zentrifugale Schenkel des Reflexbogens einzig und allein durch die erhaltene VI. lumbale Vorderwurzel repräsentiert ist. Erfolgt daher bei einer derartigen Wurzelauswahl nebst oder anstatt der reflektorischen Zehenbeugung dennoch eine reflektorische Dorsalflexion (bei leichter Reizung der Planta), so ist damit erwiesen, dass selbst die weitere Verschiebung der zentripetalen von der zentrifugalen Wurzel einen segmentären Reflex (bei leichtester Reizung) nicht zu alterieren braucht. Tatsäch-

1) Bikeles und Gizelt, l. c. 
lich überzeugten wir uns von dem Zustandekommen einer reflektorischen Dorsalflexion im Sprunggelenke selbst unter solchen Umständen.

Das Endergebnis dieser Untersuchungen lässt sich folgendermaassen ausdrücken: Die langen, bis an die motorischen Vorderhornzellen heranreichenden Hinterstrangskollateralen mögen wohl bezüglich segmentärer Reflexe die direkten Wege für die Reizübertragung auf motorische Rückenmarkselemente darstellen. Daneben existieren aber reichliche zum Erzeugen selbst dieser Reflexe auch bei minimalster Reizung an sich vollständig ausreichende indirekte Wege auf der Bahn kurzer im Hinterhorn verbleibender Hinterstrangskollateralen und deren hypothetischer Fortsetzung, sei es vermittels Zellen vom Golgi'schen Typus, sei es vermittels eingeschalteter kurzer Nervenfasern. Das Vorhandensein sowohl direkter als auch unterbrochener Bahnen zwecks einer und derselben Funktion ist eine im Zentralnervensystem vielfach bestätigte Tatsache.

\section{Prot. Dr. X. Trardovikl}

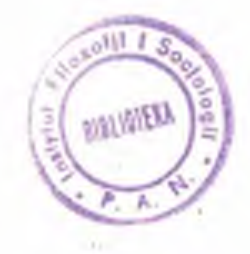

